\title{
International Journal of Innovative Research in Science, Engineering and Technology
}

(An ISO 3297: 2007 Certified Organization)

Vol. 4, Issue 3, March 2015

\section{Proof of Concept of DiffServ-aware MPLS Traffic Engineering: A VoIP-based approach}

\author{
AymenBouzid $^{1}$, AymenZelfani $^{2}$ \\ University Teacher, Department of Information Technology, ISET Nabeul, Nabeul, Tunisia ${ }^{1}$ \\ U.G. Student, Department of Telecommunication Systems Engineering, ESPRIT, Al Ghazala, Ariana, Tunisia ${ }^{2}$
}

\begin{abstract}
DS-TE is a hybrid technology that combines MPLS-TE's tunnelling capabilities with QoS-DS's guaranteed service specifications and techniques. In this paper, DS-TE's building blocks, MPLS-TE and QoS-DS, are compared one against the other in order to pinpoint the basic differences and if possible the similarities between both technologies in order to better assimilate the addition brought in by the development of DS-TE. Based on the probing and simulation capabilities of Cisco's IP SLA conducted on three topology scenarios running one of these technologies at a time, the concept validity and importance of DiffServ-aware Traffic Engineering is proven and clarified. However, the testing scenarios will be solely oriented towards the case of VoIP traffic cases.
\end{abstract}

KEYWORDS: DS-TE, QoS-DS, MPLS-TE, IP SLA, Proof of Concept.

\section{INTRODUCTION}

Delivering user-satisfactory services have always been the goal of networking service providers. To achieve credibility and promote its market presence, any service provider would try to use the best networking technologies available. One way of achieving this goal can be done by the means of quality of service (QoS) techniques which help ensure special treatments for the types of data flows that require special channeling conditions such as low delay for the case of gaming and VoIP calls. Another way applies the additions brought by packet switching in MPLS by implementing endto-end MPLS-TE tunnels in order to assign special transfer rules that help promise a certain amount of bandwidth to a specific transmission instance or flow. However, QoS wastes some amount of bandwidth upon its reservation and MPLS-TE the bandwidth reservation cannot be done for different class of services. The evolution is supposed to be brought by DiffServ-aware MPLS-TE which combines techniques from both QoS and MPLS-TE in order to deliver a better experience by providing guaranteed bandwidth services through the use of sub pools.

The work in this paper is divided in two stages. 1) Configuration and Testing 2) Analysis and interpretation.Configuration is done by simply implementing similar value-oriented configurations into the three topology cases that pertain to one of the three analyzed technologies. Those cases are then tested by the means of IP SLA to draw out the scores and calculations that could form the basis of further analysis. Once a suitable database is obtained, the results of the testing phase are plotted against one another to ease the visual and technical analysis of the similarities and differences between those technologies. Interpretations can then be made in order to prove the use and validity of DS-TE.

This paper is organized as follows. Section II describes the related technologies that constitute the basic comparison reference upon which depends DS-TE presented in Section III. After the testing tool and mechanism are introduced in Section IV, the obtained results are analyzed in Section V to discuss the technological differences and intended technical additions in DS-TE. Finally, the conclusion is presented in Section VI.

\section{RELATED TEChNOLOGIES}

a. QoS-DS

QoS(Quality of Service) comes in multiple forms such as MPLS EXP, layer 2, layer 3, and even layer 1 by prioritizing traffic. It is a filter-like technique used to assign special behaviors over the network for certain types of 


\title{
International Journal of Innovative Research in Science, Engineering and Technology
}

\author{
(An ISO 3297: 2007 Certified Organization)
}

\section{Vol. 4, Issue 3, March 2015}

traffic that usually need unique requirements such as audio for example which needs continuous data flow and low latency (among other things). The most important thing about QoS is that it is only as good as the network's weakest link. This means that, in order to get it work as optimally as possible, it is necessary to get it to work end to end. If there is a place that is not marked, the packets passing through it are the ones that will be dropped in the network. QoS offers two variants. IntServ (Integrated Services) which relies on per-flow techniques, end-to-end architecture, and shows offers scalability. The second type is DiffServ which relies on per-aggregation techniques, domain-wide service scope, and offers high scalability promises.

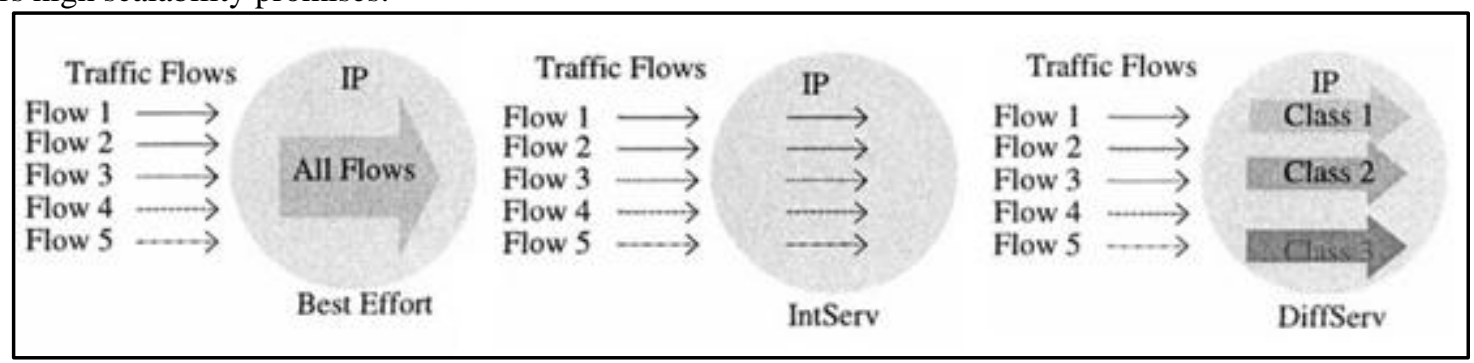

Figure 1. Traffic flow methodologies

The main idea behind Differentiated Services (DiffServ) is to provide a different set of treatments for different types of traffic in a certain networking setup.Different grades or classes of network service are defined to differently deal with different kinds of traffic which receive a treatment that could be better than others or worse depending on their defined priority and their needs. To complete the picture, all is left to be done is map the traffic from different applications to the right kind of service. As shown in figure 1, this is not available using IntServ which applies QoS by flow as a whole with no apparent relation to different types of information transferred within. The case that is even less convenient for Best Effort scenarios which only looks at transfers as a whole with no differentiation. [6]

\section{b. MPLS-TE}

MPLS-TE (Traffic Engineering using MPLS) offers the needed tool to redirect the traffic through the network in a pre-defined manner through the establishment of a logical interface called tunnel.There are two ways to define a tunnel but can be semantically extended to three. The Dynamic tunnel is the method where MPLS-TE is always enabled but only goes into action when the network needs it. Moreover, the route choice is selected automatically using PCALC with no user needed to specify any rules. The Explicit tunnel is the manual type of tunnel definition. An MPLS-TE tunnel can be created between any pair of machines in network. However, for the case of a core network or VPN, it has to be done between PEs (Provider Edges). It is very similar to static routing with the major difference residing in the fact that only runs on the tunnel's head end. To reach its destination, the tunnel needs a hop-by-hop explicit route. The Multiple pathis the final method which is simply the combination of both previous types with an introduction of a preference rule so that one picks up when the other fails.MPLS-TE relies on RSVP to define the bandwidth assigned to the logical Tunnel interface in order to reserve the needed resources. [4]

\section{DIFFSERV-AWARE TrAFFIC ENGINEERING}

DS-TE is basically an extension of the old MPLS-TE that allowed the incorporation of some QoS-DS techniques. the first thing to be encountered and noticed when using DS-TE is the introduction of a technique that allows the assignment of guaranteed bandwidth which is called BC or sub pooling. Even though DS-TE has some QoS-DS features in it, it is still a feature labeled as completely 'control plane' while QoS-DS functions on a 'data plane' type of mechanisms. [3] 


\section{International Journal of Innovative Research in Science, Engineering and Technology}

(An ISO 3297: 2007 Certified Organization)

Vol. 4, Issue 3, March 2015

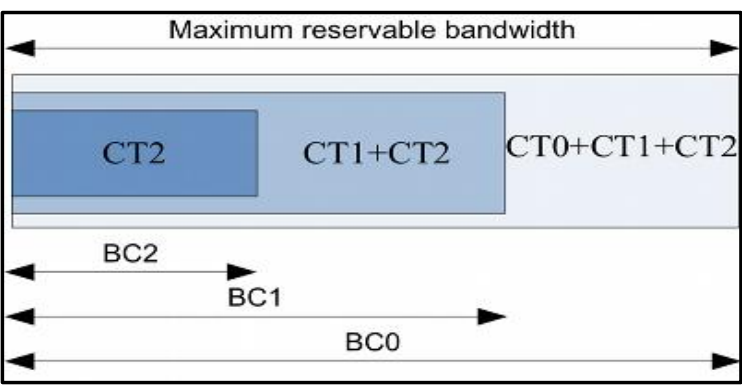

Figure 2.RDM

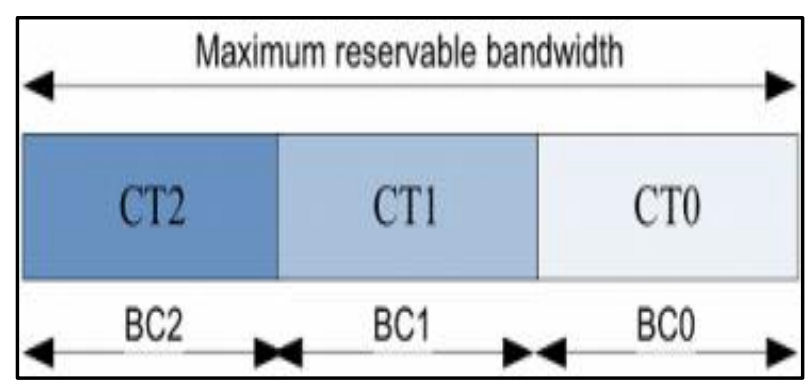

Figure 3. MAM

DS-TE uses one of two models to reserve bandwidth for different class types (CT): Maximum Allocation Model (MAM) and Russian Doll Model (RDM). MAM is the simplest bandwidth constraint model (BC) as it assigns a separate bandwidth to each CT(figure3). However, as shown in figure 3, this lacks the ability to reassign unused bandwidth to other CT. Figure 2 shows how RDM works. In this model, the bandwidth set for CT0 is used by CT0 through CT2 while the part allocated for CT2 is only used by $\mathrm{CT} 2$. This means that $\mathrm{BC} 0>\mathrm{BC} 1>\mathrm{BC} 2 \ldots$ etc. The rule here is the higher the priority the stricter the allocation meaning that CT2 in this case is given to demanding traffic such as QoS. RDM can handle up to 8 CTs ranging from 0 to 7. [1, 2]

\section{TESTING WITH IP SLA}

IP SLA (IP Service Level Agreement) is a set of features embedded in all Cisco IOS software with the exception of a few limitations for the case oflayer 2 switches. IP SLA is considered to be one of the most instrumental features in a Cisco system. What IP SLA does in summary is generate, receive, and thenanalyses certain types of traffic. For the case of UDP jitter (VoIP), IP SLA offers calculations pertaining to characteristics such as MOS, ICPIF, delay, and packet loss...etc. The testing procedure is follows the logic shown in figure 4. [5]

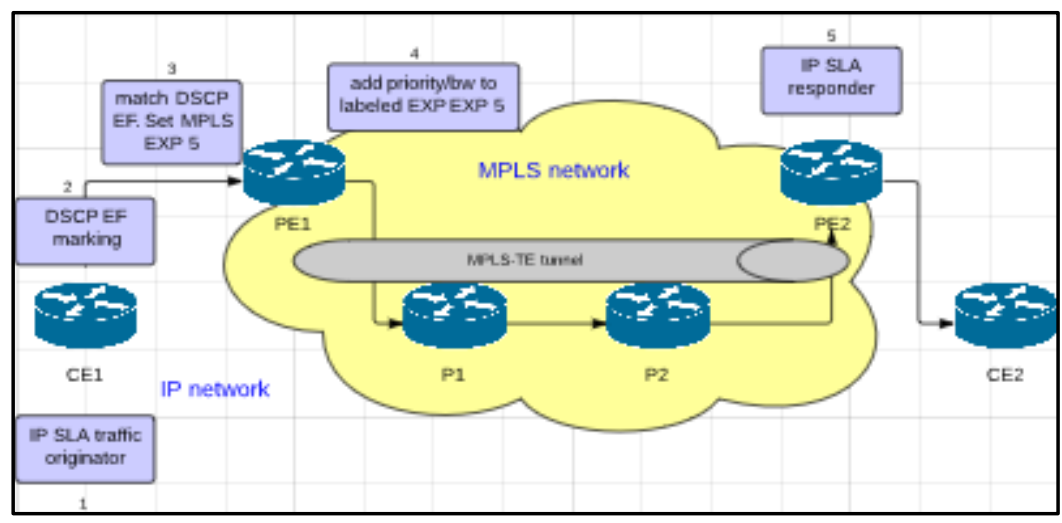

Figure 4. Testing process

A CE1 (customer edge) router initiates an IP SLA UDP jitter traffic which emulates a VoIP signal. In its outgoing interface, these packets are marked with the high priority EF DSCP code. Upon their reception at the PE1 ingress side, DSCP EF packets are matched and imposed on the MPLS network with a priority of 5 on the EXP field. The egress side adds priority or bandwidth then pushes the VoIP signals into the tunnel's designated sub pool. Once the traffic reaches the end of the tunnel (destination), IP SLA responder which is active there sends feedback to the point of origin for statistics and calculations (another tunnel is needed to send back information). The codec-size attribute is fixed at 8192 bits while the packet-number is varied from 1 to 10000 with periodical probing $(1,2,5,10,20,50,100,200,500$, 1000..). 


\section{International Journal of Innovative Research in Science, Engineering and Technology}

(An ISO 3297: 2007 Certified Organization)

Vol. 4, Issue 3, March 2015

V. RESULTS AND ANALYSIS

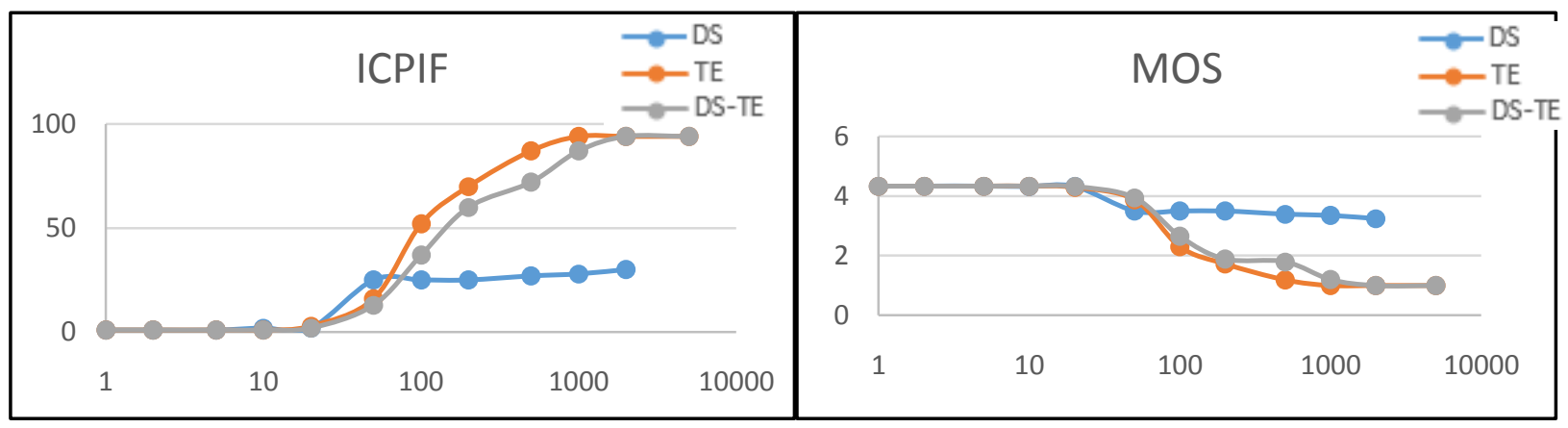

Figure 5.ICPIF comparison

Figure 6. MOS comparison

ICPIF(Impairment / Calculated Planning Impairment Factor) is one of the factors used to rate the standings of and simulated VoIP traffic. Figure 5 shows MPLS-TE and QoS-DS's ICPIF value variations with a number of packets ranging from 1 to 5000. Both configurations show close for low packet numbers which resulted in ICPIF values ranging from 1 to 5 which maps out to very good speech communication quality. For values ranging from 20 to less than 100, the DS ICPIF values fluctuated a bit pushing its plot above that of MPLS-TE [8]. This could be a first reaction to the forced congestion we are trying to achieve but it does not exactly satisfy the expected behavior of QoS. In that range, both values reached heights neighboring 25 maximum which still delivers, as analyzed by the ICPIF score, an adequate-to-good speech communication quality. The difference between both architectures starts to really show for higher numbers of packets and continue to spread apart as those numbers dramatically increase. MPLS-TE reaches values higher than 55 which would trigger high user dissatisfaction rates while QoS-DS stays under 30 which is relatively acceptable.

As opposed to how its name suggests, DS-TE is more MPLS-TE than it is QoS-DS. That is why it functions purely on the control plane while QoS-DS acts as a data plane feature. This means that DS-TE is more concerned with drawing the network map even though it has the ability to assign preferential treatment for certain packets of high QoS credentials. DS-TE does indeed show better results than MPLS-TE but still does not come close to those of QoS. As figure 5 shows, DS-TE's plot only shows some difference in the packet number range of 100 to 2000.

MOS is an acronym that stands for 'Mean Opinion Score'. It is calculated over IP SLA to simulate the quality that customers are getting. For the number of packet injection ranging from 1 to 20 (see figure 6), both MPLS-TE and QoS-DS deliver very close experiences to the users which generated the subjective response measured as a MOS value that kept staying close to 4.3. This value means that there is barely any impairment felt by the users but it is not worth mentioning or enough to complain about as it is thinly perceivable. Getting closer to 100, MOS values for both configurations showed a slight but sudden fluctuation that dropped the score to a bit below 4 for MPLS-TE which is still good while it reached 3.5 for QoS-DS which shows that users are still okay with the service but are perceiving a slightly annoying impairment. Farther into the plot, the real difference between both implementations starts to show. For packet numbers going as high as 2000, QoS-DS kept its MOS score higher than 3 which means that users are perceiving some quality impairment but that inconvenience is only slightly annoying. However, MPLS-TE's scores reach rock bottom with a very bad value of 1 which just means that the assistance team would be receiving complaints all day until these conditions change. The behavior of DS-TE judged by its tested MOS value variations shows very similar results to the ones shown and explained for ICPIF. Figure 6 still shows a very similar plot variation to those of MPLS-TE with relatively better results that are hypothetically a few steps towards QoS-DS's due to the QoS filter-like configuration set up at the tunnel's head end. However, QoS-DS is still a lot better in this regard 


\section{International Journal of Innovative Research in Science, Engineering and Technology}

(An ISO 3297: 2007 Certified Organization)

Vol. 4, Issue 3, March 2015
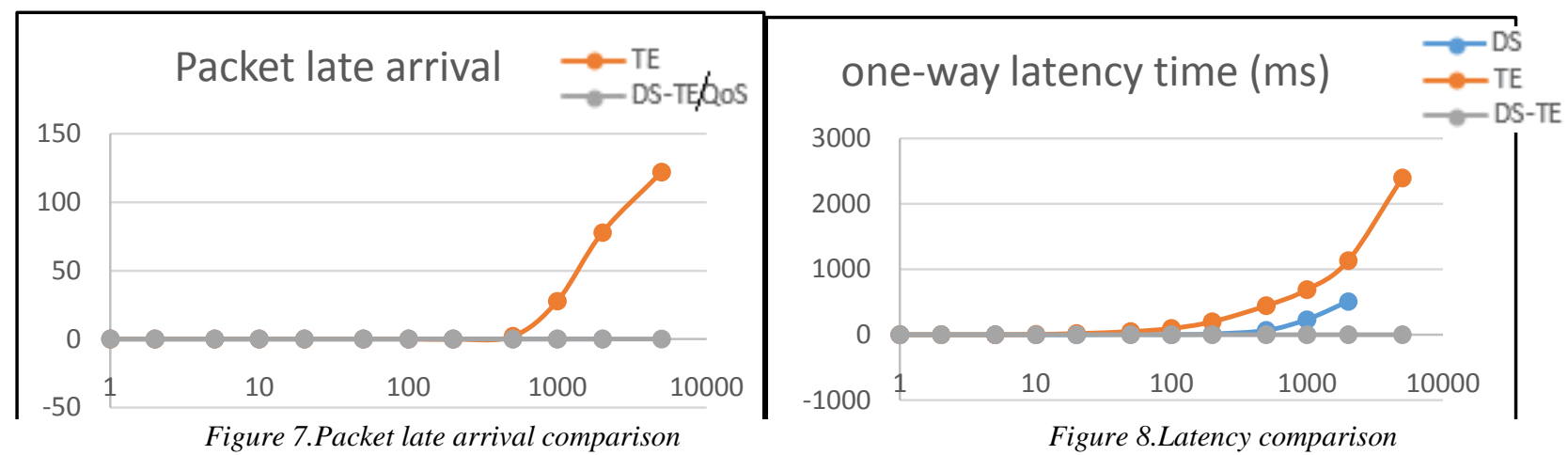

A packet is considered too late (figure 7) when it certainly did arrive but was so late that the underlying application thought it has been dropped or for the least of cases not useful anymore. For the case of VoIP which we are basing these tests on, if a packet arrives a lot later than we expect it to, the conversation keeps going thus that left-behind packet is considered too late. In the end, it is considered as bad as a drop. The first thing to be noted is QoS's behavior as it shows no signs of late arrivals whatsoever. The policy-driven techniques in QoS-DS are specifically made to allow high-priority traffic types to arrive well on time. Given that the IP SLA audio generated traffic is set to be of high precedence, no signs of packet late arrival are found within the boundaries of our test values. However, TE, shows a different behavior. Even though it remained nil for the most part of the test, it faltered at the 500 packet limit and went up high enough to reach 120 in the extreme case of 5000 audio packets. In reality, QoS can allow a few late arrivals but this test's limited scenarios have shown none. Although primarily designed over MPLS-TE, DS-TE still shows some signs of the addition of some QoS features. In figure 7, while MPLS-TE starts to exponentially rise at the mark of 500 packet number, DS-TE stays null matching the exact same behavior of QoS-DS. The reason is because how DS-TE is implemented. Before traffic starts being channeled through the tunnel, QoS rules and markings are applied on the wanted packets that are sent over the assigned tunnel with its respective sub pool. This means that the traffic inside the tunnel, in reality in the sub pool portion of the tunnel, are only the ones promised to the user to be optimized. Then, instead of optimizing traffic node by node, it is done at the tunnel entrance level then cleanly sent onto it.

For low number of packets generated by IP SLA and up to 50, both the TE and DS topologies show very low SD-latency (Source-to-destination latency) which is almost ideal. Starting from 100 and higher, both show exactly the same plot movement towards higher latency times but with different slopes. As expected, MPLS-TE generates around twice as much latency as QoS-DS. Usually, all the traffic load patterns of the network must be known to get the most out of MPLS-TE which usually auto-selects paths that may have better latency and more bandwidth. However, we are forcing both configurations to function on exactly the same path bringing into light their structural differences and raw capabilities. What is obvious here then is that TE is very sensitive to latency as it instantly fails when a lot of packets need to be transferred. QoS-TE' is different however as due to the fact that it is configured to manage VoIP packets, it recognizes that it is supposed to prioritize and transfer delay-sensitive packets which performance is easily affected by this delay. That explains why QoS-DS keeps delay times as low as possible which is consistently better than the conditions seen under MPLS-TE. Figure 8 shows the most visible difference DS-TE appears to offer throughout all our testing scenarios. It completely eliminates latency. This does not mean that DS-TE always has a similar behavior but, within the definitions and constraints of the testing scenario built around IP SLA UDP jitter simulation, it does.

It has been noted before that QoS follows the same plot fashion as TE but with lower values and a later response which allows QoS-DS to deliver a better service making it live up to the expectations of such technology. DS-TE, however, pushes those limits even more delivering a much better user experience. This is explained by the fact that, with QoS or MPLS-TE only, it is not possible to provide queue bandwidth while DS-TE can use this feature of guaranteed bandwidth using sub pools.

The only limitation to be considered is the fact that only using one type of traffic (EF-marked) limits the outcome of DS-TE's application as better results can be observed by comparing different service classes, but this work's choice has been set on VoIP traffic only which explains DS-TE's behavior being very close to MPLS-TE. 


\section{International Journal of Innovative Research in Science, Engineering and Technology}

(An ISO 3297: 2007 Certified Organization)

\section{Vol. 4, Issue 3, March 2015}

\section{CONCLUSION}

We have conducted comparative analyses on the exact same topologies running QoS-DS then MPLS-TE which have shown the technological differences between the quality-oriented QoS and the data quantity-oriented TE. The second phase covered the comparison of these two technologies with the evolved DS-TE in order to point out the addition brought by it in comparison to each of its two predecessors. In summary, the tests have shown that DS-TE is almost fully MPLS-TE but with an awareness to QoS-DS that has helped improve traffic transmission quality in terms of various specifications, especially delay.

\section{REFERENCES}

[1] Le Faucheur, F. IETF RFC 4127: Russian Dolls Bandwidth Constraints Model for Diffserv-aware MPLS Traffic Engineering. 2005

[2] Le Faucheur, F. and W. Lai. IETF RFC 4125: Maximum Allocation Bandwidth Constraints Model for Diffserv-aware MPLS Traffic Engineering. 2005.

[3] Sing, A. and Mittal, G. QoS and Traffic Engineering: MPLS, DiffServ and Constraint Based Routing. Guwahati: Department of Computer Science \& Engineering Indian Institute of Technology, pp.11-14, 2000

[4] Eric Osborne and Ajay Simha,Traffic Engineering with MPLS. Cisco Press, ISBN: 1-58705-031-5 pp.150-162, 2002

[5] Moreno Musardo “Working with cisco IP SLA: Measuring IPSLA in enterprise networks", VDM Verlag Dr. Müller. 978-3-6392-2613-3, 2010

[6] Santiago Alvarez, "QoS for IP/MPLS Networks", Cisco Press.ISBN:1587143917, 2012

[7] Alidadi A., Mahdavi M., Hashmi M.R., "A New Low- Complexity QOS Routing Algorithm for MPLS Traffic Engineering”, Proceedings of the 2009 IEEE 9th Malaysia International Conference on Communications 15-17 December 2009 Kuala Lumpur Malaysia, Pg 205- 210.

[8] Kotti, A. Hamza, R. Bouleimen, K,"Bandwidth Constrained Routing Algorithm for MPLS Traffic Engineering”, Networking and Services, ICNS, Third International Conference on, 19-25 June 2007 